\begin{tabular}{|c|c|}
\hline 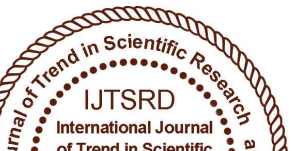 & $\begin{array}{l}\text { International Journal of Trend in Scientific } \\
\text { Research and Development (IJTSRD) }\end{array}$ \\
\hline $\begin{array}{lll} & \\
0\end{array}$ & International Open Access Journal \\
\hline and & ISSN No: $2456-6470$ | www.ijtsrd.com | Volume - 2 | Issue -3 \\
\hline
\end{tabular}

\title{
Speech Disturbance in Bilingual Female Alzheimer's Disease Patients
}

\author{
Parvaiz Ahmed Shah \\ Post Graduate, Department of Medicine, Govt. \\ Medical College, Srinagar, J \& K, India
}

\author{
Deeba Aazfa \\ Department of Linguistics, University of Kashmir, \\ Hazratbal, Srinagar, J \& K, India
}

\section{ABSTRACT}

Dementia of Alzheimer's Type is a common disorder with a massive impact on the quality of life of patients and their caregivers. After age 65 years, the prevalence of dementia increases greatly with age (Evans DA, 1989; Rocca WA, 1986). For this study, thirty Kashmiri Female Bilingual AD (MildModerate-Severe) patients and an equal number of controls matched for age, gender and education were tested in a simple Sentence Translation Task. The study aimed to find out the effect of Alzheimer's disease on the patient's ability to use syntactic information in sentence comprehension and whether the subjects are able to read and translate sentences properly. The results from the Cross-sectional Comparison in this study reveal that Mild and Moderate AD patients produce more errors in reading, comprehension and translating sentences than Control group. While as Advanced AD patients show a severe impairment in their ability to process Syntactic information.

Keywords: Dementia, Alzheimer's disease (AD), Sentence, Bilingual, Cross-sectional Comparison

\section{BACKGROUND}

Dementia of Alzheimer's Type is a common disorder with a massive impact on the quality of life of patients and their caregivers. After age 65 years, the prevalence of dementia increases greatly with age (Evans DA, 1989; Rocca WA, 1986). Older age is a well-known risk factor for AD. Fratiglioni Laura, et al., (1997) determined the incidence of different types of dementia in the very old, and explored the relation with age and gender. He has found that incidence of dementia increases with age, and women have a higher risk of developing dementia than men, especially at very old ages. Huge numbers of studies have investigated many genetic and environmental risk factors with proposed causes of AD. Family history of $\mathrm{AD}$ is one of the most strongly supported risk factor and other dementias, which has been associated with AD in large scale of population. Ferini-Strambi L et al., 1990;Fratiglioni L et al., 1993; Heyman A et al., 1984; van Duijn CM et al., 1992).Sedentary life style factors such as poor diet, drinking and less exercise are also responsible for the cause of AD.

Daniel Kempler et al., (1987) evaluated language ability of 20 patients with probable Alzheimer's disease (AD). The analysis of spontaneous speech revealed a normal range and frequency of syntactic constructions but poor lexical use. It was found that a writing task showed a similar divergence, with the ability to use syntactic cues significantly more intact than the ability to use semantic cues. The results indicated that syntactic ability is selectively preserved in AD.

In a study of Claudia Bickel et al., (2000) syntactic comprehension of German patients with dementia of the Alzheimer type was investigated and compared to healthy controls matched with respect to age, sex, and education. Syntactic structures were given the special attention. It was found that Syntactic complexity ranged from simple sentences to more complex sentences like center-embedded object relative sentences. DAT patients showed a deficit in almost all 
categories as compared to their controls. Their performance was heavily influenced by the degree of cognitive impairment. Patients with mild cognitive impairment revealed only slight problems in syntactic processing, whereas patients with moderate to severe impairment did not perform well in most syntactic categories. It was found that syntactic comprehension was mildly affected in the early stages of Alzheimer's disease and was severely impaired in advanced stages.

\section{OBJECTIVE}

This paper will attempt to look at the effect of Alzheimer's disease on the patient's concept and perception of syntactic structure of spoken sentences in Kashmiri (L1) and Urdu language (L2).

\section{PATHOLOGY:}

At autopsy, the earliest and most severe degeneration is usually found in the medial temporal lobe (entorhinal/perirhinal cortex and hippocampus), lateral temporal cortex, and nucleus basalis of Meynert. The characteristic microscopic findings are neuritic plaques and NFTs. These lesions accumulate in small numbers during normal brain aging but dominate the picture in $\mathrm{AD}$.

\section{METHODOLOGY}

A random sample of thirty normal people as control group and thirty cases of clinically diagnosed Alzheimer's disease patients are considered for the present study. This paper deals with the analysis of the thirty subjects, who suffered neuro-degeneration to the different parts of the brain and were able to respond to the tests, along with the thirty subjects as normal control group. On the basis of the medical reports, all subjects under study are categorized into three groups: Mild AD, Moderate AD, and Advanced $A D$. Out of thirty cases, 12 cases were Mild AD cases, 10 were AD Moderate and 8 were Advanced $\mathrm{AD}$ patients.

\section{TEST BATTERIES FOR LANGUAGE DEFICIT:}

Since the present study is focused on Linguistic Profiling of Alzheimer's disease rather than Dementia, it was decided to perform Kashmiri and Urdu bilingual Test. These tasks were adopted and adapted in Kashmiri and Urdu language with focus on language deficit in production, comprehension, picture naming and picture recognition abilities in Urdu language.

A syntactic test includes a given set of 10 sentences which $\mathrm{AD}$ patients have to translate from Kashmiri to Urdu and vice versa. This test allows us to understand whether the $\mathrm{AD}$ patients comprehend and translate the given sentences completely or not and 2 marks are allotted to each correct response.

\section{STATISTICAL PROCEDURE}

Out of various softwares available for the statistical analysis, / SPSS (Statistical software for social sciences) is used for the statistical analysis of data. For the data analysis in present study, SPSS used. The statistical technique namely Distance Correlation is used to determine the association between the variables in the form of distances, more the distance far the variables are from each other and vice versa. Distance in case of Distance correlation is measured in terms of Euclidean distance.

\section{DISCUSSION ON DISTANCE CORRELATION RESULTS}

The distance correlation displays Bar graphs based on Case summaries and Proximity matrix of Syntactic Test:

THE SPSS OUTPUT FOR DISTANCE CORRELATION AMONG FEMALE MILD, MODERATE AND ADVANCED AD GROUPS AND DISCUSSION ON CORRELATION RESULTS Proximity matrix and Case Summaries showing Distance Correlation in Case of Kashmiri Syntactic Test Table 1: Case Summaries

\begin{tabular}{|l|l|l|l|l|}
\hline & Control Group & Mild AD & Moderate AD & Advanced AD \\
\hline Mean & 18.968 & 17.5556 & 6.125 & 0.5 \\
\hline Std. Deviation & 0.96232 & 1.04416 & 1.15728 & 0.83666 \\
\hline Time & 95 & 203 & 581 & 900 \\
\hline
\end{tabular}


Table 1 indicates that the average score taken by a Control Group in Kashmiri Syntactic test is 18.9 while as score taken by the Subjects in Mild, Moderate and Advanced Stage are 17.5, 6.1 and 0.5 respectively, also the time taken by a Control Group in this test is 95 seconds while as time taken by Subjects in Mild, Moderate and Advanced Stages are 203, 581 and 900 seconds respectively.

Table 2: Proximity Matrix

\begin{tabular}{|c|c|c|c|c|}
\hline & \multicolumn{4}{|c|}{ Euclidean Distance } \\
\cline { 2 - 5 } & Control Group & Mild AD & Moderate AD & Advanced AD \\
\hline Control Group & 0 & 4.036 & 31.237 & 45.649 \\
\hline Mild AD & 4.036 & 0 & 27.955 & 42.418 \\
\hline Moderate AD & 31.237 & 27.955 & 0 & 14.705 \\
\hline Advanced AD & 45.649 & 42.418 & 14.705 & 0 \\
\hline
\end{tabular}

Table 2 is called a Proximity matrix/Distance Matrix/Dissimilarity Matrix which is used to predict the differences in the variables, more value between the variables corresponds to the severity of the case. Whereas, 0 value indicates that variable are same.

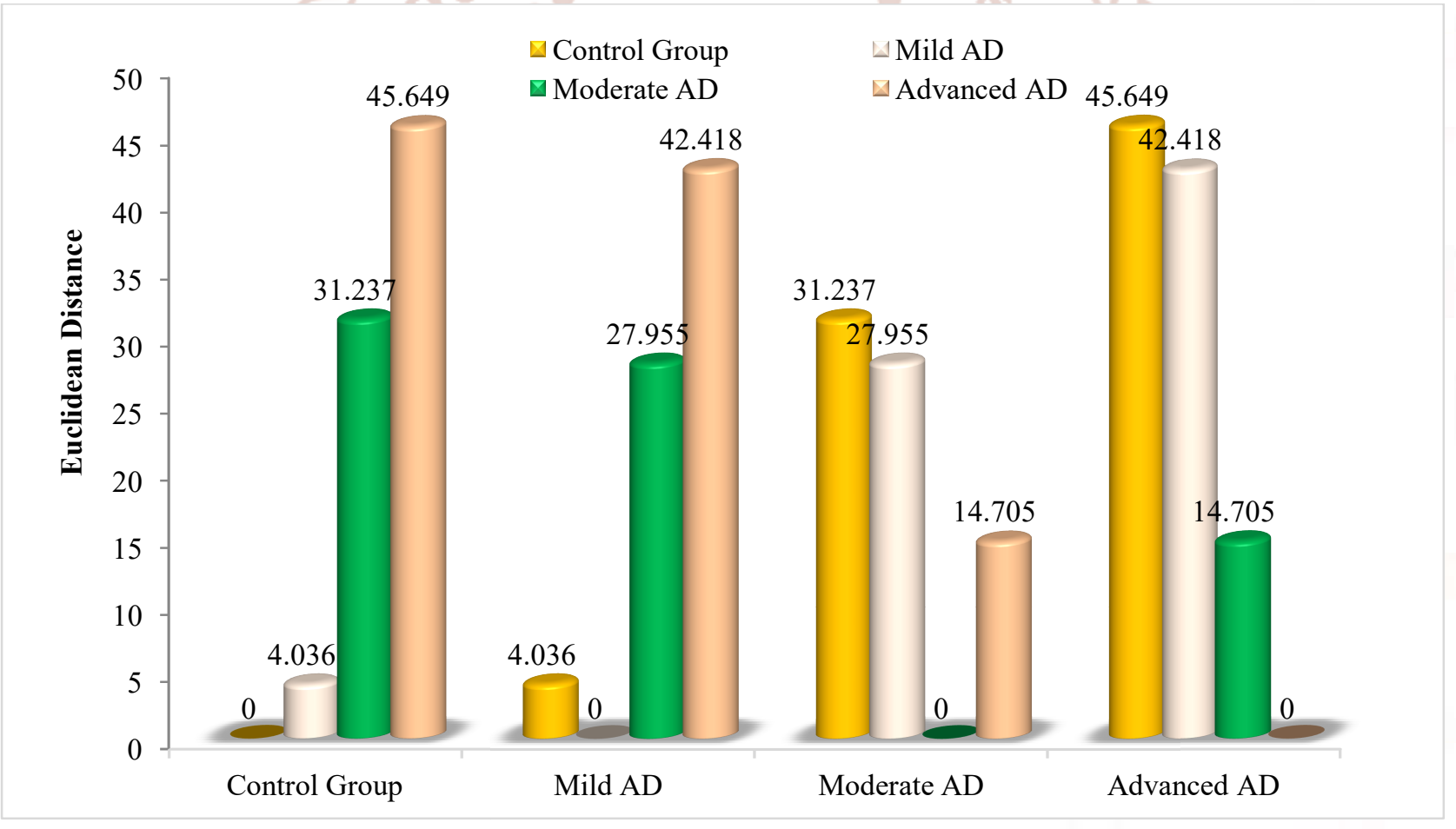

Fig 1: Euclidean distance showing difference between Mild, Moderate and Advanced Female AD Patients in case of Kashmiri Syntactic Test.

From the table and the bar chart given above, the following conclusions can be drawn:

1. The performance of Control group and Mild AD subjects is almost similar in Kashmiri Syntactic test with a minimum Euclidean distance of 4 between them as compared to Moderate $\mathrm{AD}$ and Advanced AD subjects. However, Mild AD subjects seem to be less affected but still there is an evident sentence breakdown.

2. The Moderate AD and Advanced AD subjects have apparently more disorder in translating Kashmiri sentences into Urdu.

3. As compared to Control Group, the Euclidean distance shows increase from Mild AD to Moderate $\mathrm{AD}$ and from Moderate $\mathrm{AD}$ to 
International Journal of Trend in Scientific Research and Development (IJTSRD) ISSN: 2456-6470

Advanced AD subjects. The Euclidean distance between Control Group and Mild AD subjects is 4 whereas, the Euclidean distance between Control Group -Moderate AD subjects and Control GroupAdvanced AD subjects is 31.2 and 45.6 respectively. The Euclidean distance is least for Mild AD subjects and greater for Advanced subjects.

4. As compared to Mild AD subjects, the Euclidean distance between Mild AD-Moderate AD subjects and Mild AD -Advanced AD subjects is 27.9 and 42.4 respectively.

5. As compared to Moderate AD subjects, the Euclidean distance between Mild AD-Moderate $\mathrm{AD}$ subjects and Moderate AD -Advanced AD subjects is 27.9 and 14.7 respectively.

6. As compared to Advanced AD subjects, the Euclidean distance between Mild AD-Advanced $\mathrm{AD}$ subjects and Moderate AD -Advanced AD subjects is 42.4 and 14.7 respectively.

\section{Proximity matrix and Case Summaries showing Distance Correlation in Case of Urdu Syntactic Test}

Table 3: Case Summaries

\begin{tabular}{|l|c|c|c|c|}
\hline & Control Group & Mild AD & Moderate AD & Advanced AD \\
\hline Mean & 20 & 17.1111 & 6.5 & 0.6667 \\
\hline Std. Deviation & 0 & 1.2693 & 1.30931 & 1.21106 \\
\hline Time & 69 & 186 & 404 & 900 \\
\hline
\end{tabular}

Table 3 indicates that the average score taken by a the time taken by a Control Group in this test is 69 Control Group in Urdu Syntactic test is 20 while as seconds while as time taken by Subjects in Mild, score taken by the Subjects in Mild, Moderate and Moderate and Advanced Stages are 186, 404 and 900 Advanced Stage are 17, 6.5 and 0.6 respectively, also seconds respectively.

Table 4: Proximity Matrix

\begin{tabular}{|l|l|l|l|l|}
\hline \multirow{2}{*}{} & \multicolumn{2}{|l|}{ Euclidean Distance } \\
\cline { 2 - 5 } & Control Group & Mild AD & Moderate AD & Advanced AD \\
\hline Control Group & 0 & 6.164 & 31.906 & 47.434 \\
\hline Mild AD & 6.164 & 0 & 26.325 & 41.881 \\
\hline Moderate AD & 31.906 & 26.325 & 0 & 16.062 \\
\hline Advanced AD & 47.434 & 41.881 & 16.062 & 0 \\
\hline
\end{tabular}

Table 4 is called a Proximity matrix/Distance Matrix/ Dissimilarity Matrix which is used to predict the differences in the variables, more value between the variables corresponds to the severity of the case. Whereas, 0 value indicates that variable are same. 


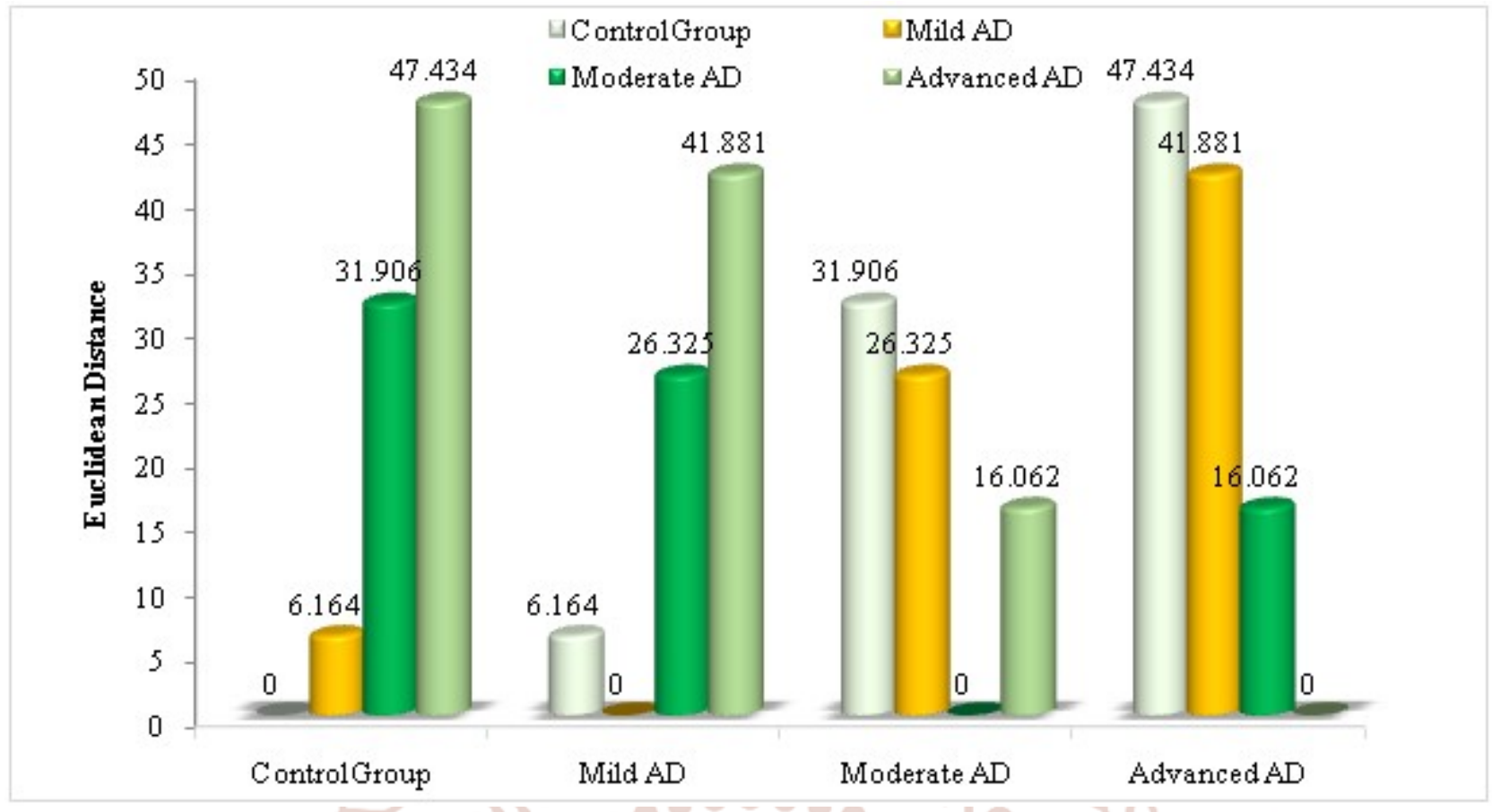

Fig 2: Euclidean distance showing difference between Mild, Moderate and Advanced Female AD Patients in case of Urdu Syntactic Test

From the table and the bar chart given above, the following conclusions can be drawn:

1. The performance of Control group and Mild AD subjects are almost similar with a minimum Euclidean distance of 6 between them in Urdu Syntactic test. Hence, the Mild AD subjects have satisfactory control over Sentence reading and comprehension.

2. However, in case of Advanced AD subjects there is a drastic fall of result which leads to abrupt increase in Euclidean distance. The Moderate AD and Advanced AD subjects have apparently more disorder in translating Urdu sentences into Kashmiri. The difference is quite obvious.

3. As compared to Control Group, the Euclidean distance shows increase from Mild AD to Moderate $\mathrm{AD}$ and from Moderate $\mathrm{AD}$ to Advanced AD subjects. The Euclidean distance between Control Group and Mild AD subjects is 6.1 whereas, the Euclidean distance between Control Group -Moderate AD subjects and Control Group-Advanced AD subjects is 31.9 and 47.4 respectively. The Euclidean distance is least for Mild AD subjects and greater for Advanced subjects.
4. As compared to Mild AD subjects, the Euclidean distance between Mild AD-Moderate AD subjects and Mild AD -Advanced AD subjects is 26.3 and 41.8 respectively.

5. As compared to Moderate AD subjects, the Euclidean distance between Mild AD-Moderate $\mathrm{AD}$ subjects and Moderate $\mathrm{AD}$-Advanced $\mathrm{AD}$ subjects is 26.3 and 41.8 respectively.

6. As compared to Advanced AD subjects, the Euclidean distance between Mild AD-Advanced $\mathrm{AD}$ subjects and Moderate $\mathrm{AD}$-Advanced $\mathrm{AD}$ subjects is 41.8 and 16 respectively.

\section{CONCLUSION}

Women are more prone to Alzheimer's disease as compared to males. The analysis of spontaneous speech revealed a normal range and frequency of syntactic constructions but poor lexical use. As the AD patient suffers from anomia, it becomes difficult for them to retrieve the word which ultimately leads to sentence breakdown. In comparison to their controls, patients show a mild to severe deficit as we move from Mild to Advanced group. The results indicated that syntactic ability is selectively preserved in AD. It was found that syntactic comprehension was mildly affected in the early stages of Alzheimer's 
disease and was severely impaired in advanced stages. Patients with mild cognitive impairment, showed only slight difficulties in syntactic processing, whereas patients with moderate to advanced impairment showed severe difficulties. Moreover, results also show that female AD subjects show better performance in Urdu Syntactic Test as compared to Kashmiri Syntactic Test.

\section{REFERENCES}

1. Bialystok, Ellen, Fergus IM Craik, and Morris Freedman. "Bilingualism as a protection against the onset of symptoms of dementia."Neuropsychologia, Vol. 45, no.2, 2007, pp. 459-464

2. Bickel, Claudia, et al. "Syntactic comprehension deficits in Alzheimer's disease." Brain and Language, vol. 71, no.3, 2000, pp. 432-448

3. Evans, Denis A., et al."Prevalence of Alzheimer's disease in a community population of older persons: Higher than previously reported." Journal of the American Medical Association, vol. 262, Nov. 1989, pp. 2551-2556

4. Fratiglioni, Laura, et al. "Risk factors for late-onset Alzheimer's disease: A population-based, case-control study." Annals of neurology, vol. 33, no.3, 1993, pp. 258-266
5. Fratiglioni, Laura, et al. "Very Old Women at Highest Risk of Dementia and Alzheimer's Disease Incidence Data from the Kungsholmen Project, Stockholm." Neurology, vol. 48, no.1, 1997, pp. 132-138

6. Heyman, Albert, et al. "Alzheimer's disease: A study of epidemiological aspects." Annals of neurology, vol. 15, no.4, 1984, pp. 335-341

7. Hyltenstam, Kenneth, and Loraine K. Obler, eds. Bilingualism across the lifespan: Aspects of acquisition, maturity and loss. Cambridge University Press, 1989.

8. Kempler, Daniel, Susan Curtiss, and Catherine Jackson. "Syntactic preservation in Alzheimer's disease." Journal of speech and hearing research, vol. 30, no.3, 1987, pp. 343-350.

9. Rocca, W. A., L. A. Amaducci, and B. S. Schoenberg. "Epidemiology of clinically diagnosed Alzheimer's disease." Alzheimer Disease \& Associated Disorders, vol. 1, no.2, 1987, pp. 105.

10. Van Duijn, Cornelia M., and A. Hofman. "Risk factors for Alzheimer's disease: the EURODEM collaborative re-analysis of case-control studies."Neuroepidemiology, vol. 11, suppl. 1, 1992, pp. 106-113. 